\title{
Correction to: Chinese secondary school students' reading engagement profiles: associations with reading comprehension
}

\author{
Jiangze Lin ${ }^{1} \cdot$ Qian $\mathrm{Li}^{2} \cdot$ Haoqi Sun ${ }^{2} \cdot$ Zhijun Huang $^{3} \cdot$ Guomin Zheng ${ }^{1}$
}

Published online: 7 June 2021

(c) Springer Nature B.V. 2021

\section{Correction to: Reading and Writing https://doi.org/10.1007/s11145-021-10139-4}

In the original publication of the article, the Appendix section was erroneously included. This has been removed with this Correction.

The original article has been corrected.

Publisher's Note Springer Nature remains neutral with regard to jurisdictional claims in published maps and institutional affiliations.

\begin{abstract}
All authors contributed to the study conception and design. Guomin Zheng is the correspondingauthor, Jiangze Lin and Qian Li are the co-first authors of the article. Materials preparation, datacollection, and analysis were performed by Jiangze Lin. The first draft of the manuscript was writtenby Jiangze Lin, Qian $\mathrm{Li}$, and Haoqi Sun, and all authors commented on previous versions of themanuscript. All authors read and approved the final manuscript.
\end{abstract}

The original article can be found online at https://doi.org/10.1007/s11145-021-10139-4.

\author{
Guomin Zheng \\ zhengguomin_bnu@163.com \\ Jiangze Lin \\ jiangzelin@mail.bnu.edu.cn \\ Qian Li \\ liqian1008bnu@hotmail.com \\ Haoqi Sun \\ haoqi_sun@mail.bnu.edu.cn \\ Zhijun Huang \\ zjhuang@ @ese.ecnu.edu.cn
}

1 School of Chinese Language and Literature, Beijing Normal University, Xin Jie Kou Wai Street \#19, Beijing 100875, People's Republic of China

2 Collaborative Innovation Center of Assessment for Basic Education Quality, Beijing Normal University, Xin Jie Kou Wai Street \#19, Beijing 100875, People's Republic of China

3 Department of Special Education, Faculty of Education, East China Normal University, Zhong Shan Bei Road \#3663, Shanghai 200062, People's Republic of China 